\title{
Soil organic matter and ammonium affect potassium retention in soil microaggregates under long-term fertilization
}

\author{
Mingjun Chen ${ }^{1}$, Xiaoqin Chen ${ }^{2}$, Shahbaz Muhammad ${ }^{3}$, Tida Ge ${ }^{4}$, Huoyan Wang ${ }^{5}$, Shu \\ Wang $^{1}$, and Wenzhao Zhang ${ }^{6}$ \\ ${ }^{1}$ Southwest Forestry University \\ ${ }^{2}$ Institute of Soil Science Chinese Academy of Sciences \\ ${ }^{3}$ Centre for Environmental and Climate Science \\ ${ }^{4}$ Institude of Subtropical Agriculture, The Chinese Academy of Sciences \\ ${ }^{5}$ Institute of Soil Science, Chinese Academy of Sciences \\ ${ }^{6}$ Institute of Subtropical Agriculture Chinese Academy of Sciences
}

September 25, 2021

\begin{abstract}
The adsorption and fixation of potassium in agricultural soils are important as they influence K availability for crops. Soil organic matter (SOM) and ammonium (NH4+) exist in soils and play indispensable roles in soil fertility and crop yield; however, the effects of SOM and NH4+ on K retention in soil aggregate remains unclear. This study aimed to evaluate the effects of SOM and $\mathrm{NH} 4+$ on $\mathrm{K}$ adsorption and fixation in soil microaggregates $(<0.25 \mathrm{~mm})$. Soil microaggregates were extracted from three long-term fertilization treatments under rice-wheat rotations: no fertilizer (CK), fertilized with inorganic NPK (NPK), and inorganic NPK fertilizers combined with straw return (NPKS). Long-term fertilization, particularly the application of inorganic NPK combined with straw return, significantly improved the SOM content in microaggregates. Both NPK and NPKS treatments increased $\mathrm{K}$ adsorption but decreased $\mathrm{K}$ fixation, and SOM oxidation of microaggregates reduced $\mathrm{K}$ adsorption but increased $\mathrm{K}$ fixation in all treatments, indicating the positive and inhibitory effects of SOM on $\mathrm{K}$ adsorption and fixation, respectively. $\mathrm{NH} 4+$ significantly inhibited $\mathrm{K}$ adsorption and fixation, and this inhibitory effect was more significant in microaggregates with a higher SOM content. Although NH4+ reduced the positive effect of SOM on K adsorption, it enhanced the inhibitory effect of SOM on K fixation. Conclusionally, long-term fertilization increases K adsorption but reduces K fixation by improving SOM content, where $\mathrm{NH} 4+$ enhances SOM inhibited K retention in soil microaggregates, which is considered to improve K availability in soils amended with $\mathrm{K}$ fertilizers. Keywords: soil organic matter, NH4+, $\mathrm{K}$, adsorption, fixation
\end{abstract}

\section{Hosted file}

manuscript.doc available at https://authorea.com/users/436116/articles/538670-soil-organicmatter-and-ammonium-affect-potassium-retention-in-soil-microaggregates-under-long-termfertilization 